\title{
An overview of experimental results and dispersion modelling of nanoparticles in the wake of moving vehicles
}

Matteo Carpentieri, Prashant Kumar* and Alan Robins

Faculty of Engineering and Physical Sciences, University of Surrey, Guildford GU2 $7 X H, U K$

\section{Capsule}

The transformation of nanoparticles and the flow characteristics in both the near and far wake regions must be understood in order to develop mathematical models.

\begin{abstract}
Understanding the transformation of nanoparticles emitted from vehicles is essential for developing appropriate methods for treating fine scale particle dynamics in dispersion models. This article provides an overview of significant research work relevant to modelling the dispersion of pollutants, especially nanoparticles, in the wake of vehicles. Literature on vehicle wakes and nanoparticle dispersion is reviewed, taking into account field measurements, wind tunnel experiments and mathematical approaches.
\end{abstract}

Field measurements and modelling studies highlighted the very short time scales associated with nanoparticle transformations in the first stages after the emission. These transformations strongly interact with the flow and turbulence fields immediately behind the vehicle, hence the need of characterising in detail the mixing processes in the vehicle wake. Very few studies have analysed this interaction and more research is needed to build a basis for model development. A possible approach is proposed and areas of further investigation identified.

Key words: Dispersion model; Nanoparticles number concentration; Particle size distribution; Street canyons; Near and far wake; Vehicle exhaust emission

${ }^{*}$ Corresponding author. Division of Civil, Chemical and Environmental Engineering, University of Surrey, Guildford GU2 7XH, UK; Tel.: +44 1483 682762; Fax: +44 1483 682135. Email addresses: P.Kumar@surrey.ac.uk; Prashant.Kumar@cantab.net 


\section{Introduction}

Atmospheric nanoparticles have attracted substantial attention from the scientific community due to their negative impacts on human health (Donaldson et al., 2005), urban visibility (Jacobson, 2005) and global climate change (IPCC 2007). Recent toxicological and epidemiological evidence indicates that number concentration is one of the most important metrics to assess effects on human health (ICRP, 1994; Oberdorster, 2000; Davidson et al., 2005; Donaldson et al., 2005). Road vehicles are the dominant source of nanoparticles, contributing up to $\sim 86 \%$ of total particle number concentrations in the urban environment (Johansson et al. 2007; Pey et al. 2009). More than $99 \%$ of the particles, by number, in the atmospheric urban environment are in the $<300 \mathrm{~nm}$ size range (Kumar et al., 2008a-d; Kumar et al., 2009a). Exhaust emissions from vehicles can increase ambient number concentrations of nanoparticles by two orders of magnitude or more $\left(10^{4}-10^{6} \# \mathrm{~cm}^{-3}\right)$ relative to the background level $\left(10^{3}-10^{4} \mathrm{~m} \mathrm{~cm}^{-3}\right)$ (Kumar et al., 2008c, d; Kumar et al., 2009b). It is, therefore, important to examine emissions from individual vehicles under real driving and dilution conditions. However, the situation becomes complex when fine spatial scale studies of nanoparticle dispersion are contemplated (e.g. in a vehicle wake) because of the limited time response of most available instrumentation. Our preliminary results (Kumar et al., 2009c) showed that the particle number distribution and concentration of nanoparticles changes rapidly in the wake of a moving vehicle due to the influence of a number of transformation processes (i.e. coagulation, condensation, deposition and nucleation). This is because, as the exhaust dilutes and cools, volatile precursors present in sufficient concentrations may become sufficiently supersaturated to nucleate, grow and undergo gas-to-particle conversion (Kittelson et al., 2006a). This is the key difference between modelling dispersion for standard 
gaseous pollutants and nanoparticles. The very short time scales of this evolution (less than a few seconds) call for a more accurate description of the mixing process in the vehicle wake than available today.

A number of methods exist for modelling the dispersion of passive pollutants away from a roadway, though they very rarely can treat vehicle wakes or include any adjustment due to this effect; for example, CALINE4 (Benson, 1989), CPBM (Yamartino and Wiegand, 1986), ADMS-Urban (CERC, 2006) and OSPM (Berkowicz, 2000). Most of these models treat the emission as a continuous line source, either without any adjustment or by a simple enhancement of turbulence levels (Baker, 2001), despite the significant effect on the dispersion of pollutants that vehicle wakes have. There have been a modest number of studies concerning the aerodynamic behaviour of ground vehicles and the effect of ground structures on drag (Eskridge et al., 1979; Eskridge and Hunt, 1979; Baker, 2001). However, little attention has been paid to the wake properties that are connected with the dispersion of nanoparticles. On the other hand, a number of studies have been dedicated to measurements of nanoparticles along the roadside and in street canyon (Charron and Harrison, 2003; Gidhagen et al., 2004; Brugge et al., 2007; Kumar et al., 2008a; Kumar et al., 2008d; Kumar et al., 2009b). However, the processes of transport and transformation that relate these observations to vehicle tailpipe emissions remain largely unknown.

The objectives of this study are to synthesise significant past research work relevant to nanoparticle dispersion modelling in the vehicle wakes, together with highlighting associated research gaps. Section 2 presents a critical discussion of the characteristics of vehicle wakes and their mixing properties. Section 3 gives an introduction to the transformation processes of nanoparticles emitted by vehicles. The most relevant 
literature on this topic is then reviewed in the following sections, organised in three distinct categories: field measurements (Section 4), wind tunnel experiments (Section 5) and mathematical modelling (Section 6). Section 7 summarises the previous sections, identifies future research needs and sets up a research strategy for developing mathematical nanoparticle dispersion models and forming appropriate assumptions on the role of fine scale particle dynamics in existing dispersion models. Such models would assist regulatory authorities in designing sensible future actions to regulate nanoparticle emissions.

\section{Flow and mixing in the wake of moving vehicles}

A detailed knowledge of flow and mixing processes in vehicle wakes is necessary in order to cope with the very short time scales of nanoparticle evolution processes; an adequate treatment of such processes is essential for developing reliable mathematical models. In this study, we are particularly interested in the case of a moving vehicle with little or no cross-wind. When a vehicle moves forward at velocity $V$ through the undisturbed air, the flow separates from the bluff rear end in the wake. The wake as a whole may generally be divided into two distinct regions: the near wake and the main or far wake (Fig. 1). The near wake is the region of separated flow close behind the body. For a road vehicle, it is characterised by both a region of recirculation and the formation of a pair of streamwise longitudinal vortices (Hucho, 1987). The far wake is an area of general turbulence downstream of the near wake, which has little discernible flow structure (Hucho, 1987).

\subsection{Near-wake}

The near-wake of a car consists of two components: a large scale recirculation region immediately behind the vehicle and a system of longitudinal trailing vortices 
with unsteady fluctuations caused by a variety of effects such as the instability of the separated shear layer and wake pumping (Ahmed, 1981; Hucho, 1987; Baker, 2001).

The initial dispersion of the exhaust plume in the near wake becomes important at the local scale, when receptors (e.g., people) are at close distance (in the same street) as the emission source. However, the level by which this will affect the local scale dispersion depends by the local conditions. It is likely that in very dense traffic conditions and deep street canyons the near wake may be neglected, but more studies are needed to support this hypothesis.

Studies related to the characterisation of dispersion behaviour in the near-wake are much rarer than those related to the far wake zone (see Section 2.2). Baker (1996) describes a model based on the assumption that the pollutant emitted by the vehicle is spread uniformly in the near wake (that is the plume is coincident with the vehicle wake), using a Gaussian puff approach to calculate the concentrations further downwind. This approach might be acceptable for passive gaseous pollutants, but nanoparticles experience transformations on very short time scales (see Section 3) and a more detailed characterisation of the near wake may therefore be necessary. More

recently, computational fluid dynamics (CFD) have been applied to the near-wake dispersion of pollutants (Richards, 2002; Dong and Chan, 2006). The simulations, however, are limited by the lack of experimental studies specifically aimed at deriving suitable boundary conditions for the numerical calculations.

\subsection{Far wake}

The most well known and documented vehicle wake theory is that of Eskridge and Hunt (1979). Based on the perturbation analysis of the equations of motion the theory describes the velocity field far downstream of a single vehicle moving through 
still air. Using the assumptions of constant vehicle velocity, flat terrain and no wind, expressions were developed for the velocity deficit far downwind of a vehicle i.e. the far wake. A more recent study by Hider et al. (1997) saw the derivation of the same expression using a different method. They also derived expressions for the lateral and vertical velocity components.

Dispersion in the far wake is usually treated as a standard Gaussian plume (Baker, 1996; 2001; Richards, 2002). Very few models take into account the effect of the vehicle wake in the dispersion process (see Section 6).

The influence of cross-winds has also been studied. The primary effect, either in open terrain or within a street canyon, it is to translate the wake in the wind direction (Eskridge and Hunt, 1979; Yamartino and Wiegand, 1986; Hider et al., 1997; Baker, 2001). However, there are other effects due to atmospheric turbulence that are strongly dependent on the surrounding topography (open terrain or street canyon, for example; see Baker, 2001).

Table 1 summarise some of the key flow and mixing characteristics to be considered for developing a mathematical model for the vehicle wake (near and far wakes). Clearly, further research is needed for the adequate characterisation of vehicle wakes in dispersion models. In particular, almost no information can be found on the mixing process in the near-wake, though this is the key region where the nanoparticle main evolution processes occur (see Section 3).

\section{Short range dispersion of nanoparticles in the urban environment}

The behaviour of particles in the atmosphere and within the human respiratory system is determined largely, but not wholly, by their physical properties, which have 
a strong dependence upon particle size (AQEG, 2005). The smallest group of particles, with diameters of $\leq 30 \mathrm{~nm}$, are usually referred to as the nucleation mode (Charron et al., 2008; Kumar et al., 2010a, b, c). Growth of nucleation mode particles, primarily by vapour condensation but also as a result of coagulation processes, leads to the formation of accumulation mode particles that are typically between 30 and 300 $\mathrm{nm}$ in size (Kumar et al., 2010b). Particles in the accumulation mode can have long atmospheric lifetimes, typically 7-30 days in the absence of rain, much longer than the short-lived particles in the nucleation mode (Kumar et al., 2010b).

Nanoparticles emitted by cars are subject to dilution (mixing) processes very similar to those described in the previous section for passive gaseous emissions. They are, however, also subject to a number of other processes at various stages in their evolution (e.g. nucleation, coagulation, condensation and deposition). Ketzel and Berkowicz (2004) analysed the time scales associated with different transformation processes. They concluded that dilution is the fastest transformation process at most concentration levels, followed by condensation. Coagulation and deposition do not usually play a significant role in defining particle number concentrations near streets. However, they did not treat the very first stages of dispersion, where nucleation and condensation are mainly responsible for the formation of nanoparticles. Details of the interaction between dilution and other transformation processes at short distances from the emission point were studied by Zhang and Wexler (2004). They found that the exhausts emitted from different types of engines retained their own characteristics in the first stage of the dilution process. Sulphuric acid-induced nucleation was found to be the dominant particle production mechanism, followed by the condensation of organic compounds, resulting in the rapid growth of nuclei mode particles and relatively slow growth of accumulation mode particles. This study, however, did not 
include any effect due to the vehicle wake, which, as described in Section 2, strongly modifies the flow and turbulence fields near the emission source. A summary of the significance of different transformation processes is reported in Table 2; the table has been compiled using information from Ketzel and Berkowicz (2004) and the other references mentioned in this section.

\section{Field measurements}

A number of field measurements of number and size distributions of nanoparticles in urban areas have been made using instruments such as scanning mobility particle sizers, electrical low pressure impactors, ultrafine particle condensation counters, alone or in combination (Shi et al., 1999; Longley et al., 2003; Wehner and Wiedensohler, 2003; Weber et al., 2006). A comprehensive review covering this topic can be found in Kumar et al. (2010b). Most of these instruments have low sampling frequencies relative to that required to characterise nanoparticle dispersion phenomena in vehicle wakes, hence an instrument with a fast response is required (Kumar et al., 2009c). The use of one such instrument (a Cambustion DMS500) in a street canyon and a vehicle wake was recently reported by Kumar et al. (2008a-d, 2009a-c). Yao et al. (2006) confirm the need for fast response instrumentation. They measured the concentration of nanoparticles emitted by a vehicle and applied different averaging times in the range from 1 to 120 s and concluded that time-averaging data from complex environments, such as street canyons and tunnels, can affect their interpretation and lead to misleading or meaningless deductions regarding particle evolution.

Field experiments purposely designed for measuring nanoparticle evolution in the wakes of vehicles are rare and there's a clear need for further research. Kumar et al. 
(2009c) measured nanoparticle concentrations in the wakes of a moving diesel vehicle. In their preliminary experiments, a fixed measurement point was placed 20 $\mathrm{cm}$ above the ground and used to obtain nanoparticle number concentrations from plumes dispersed by diesel vehicles passing over the sampling tube. The results from this particular study are summarised in Fig. 2. An important finding was that the effects of transformation processes, such as nucleation and condensation, were generally complete within about $1 \mathrm{~s}$ after emission, showing the rapid evolution of particle number and mass distributions in the near-wake. Because of very limited observations, these preliminary results are useful for understanding the dynamics of nanoparticles at very short time scales but not sufficient to enable sensible mathematical models to be developed. There is still an need for detailed experiments using the fast response instruments to estimate the evolution time of particle number and mass distributions for various types of vehicles running at a range of speeds in different urban settings and meteorological conditions, where background particle number concentrations differ considerably. Additionally, it would be of considerable value if detailed investigations of the transformation processes were also carried out to help address important questions about the role of fine scale particle dynamics.

\section{Wind tunnel experiments}

Wind tunnel experiments have been used extensively for determining the flow characteristics of wake behind vehicles, such as Eskridge and Thompson (1982) and Hackett et al. (1987), but studies for pollutant dispersion, particularly for nanoparticles, are relatively rare. To our knowledge, there have not been any wind tunnel experiments involving nanoparticle measurements so far. Results from such studies in the controlled environment of the wind tunnel will be able to complement the data from field studies and, in conjunction with flow and tracer gas measurements, 
greatly contribute to the development of mathematical models. There have been, however, a number of wind tunnel studies that, even if not directly related to nanoparticle dispersion, provide a useful basis for the development of future experiments and mathematical models. Subsection 5.1 describes the extensive work carried out for deriving parameterisation for traffic produced turbulence. This is relevant to enhance our understanding of the mixing process in the near wake (see, for example, Table 1). The following subsection describes the few attempts at characterising dispersion behaviour in the wake of moving vehicles, in particular in the near wake. The third subsection discusses improvements in the methodologies and identifies further experimental work that can help in the development of models for nanoparticle dispersion models in vehicle wake.

\subsection{Traffic produced turbulence}

Traffic produced turbulence (TPT) and its effects on the flow and mixing processes has been the main focus of a number wind tunnel studies. For instance, Kastner-Klein et al. (2000a, b; 2001a, b) studied different traffic configurations (one-way and two-way) simulated by small metal plates moving on two belts along the street in a wind tunnel model. Their main interest was in the interactions between traffic- and wind-induced flows in a street canyon. The presence of traffic and its arrangement were also shown to affect the concentration distribution along the leeward canyon wall (Kastner-Klein et al., 2001b). The results were then used to derive TPT parameterisations for street canyon models (Kastner-Klein et al., 2003; Di Sabatino et al., 2003).

Khare et al. (2002) and Ahmad et al. (2002) used a similar approach, consisting of moving belts carrying model vehicles, in their wind tunnel experiments. The system 
was placed in various types of simulated atmospheric boundary layers, and the effect of the traffic condition and the wind direction on the vertical spread of the exhaust gas was examined. Since the main concern was the effect of traffic as a whole on the general flow and mixing processes in the urban environment, the above mentioned studies did not analyse in detail the characteristics of the wake behind a single moving element. None of them studied the near wake region of the vehicles.

\subsection{Dispersion of inert tracers in the vehicle wake}

For the development of the ROADWAY model, Eskridge and Rao (1986) determined the optimal turbulence scales in the far wake region of a moving vehicle. This was determined by measuring concentrations of inert gaseous tracer in the farwake (30-60 car-heights behind) of a full scale model of a passenger car in the wind tunnel. The near-wake was not the focus of their study, though. More relevant to the near-wake was the study conducted by Clifford et al. (1997). They used three passenger car models in a row in the wind tunnel, separated by half the car length from each other and measured the concentration distribution of the tracer on the car surface including air inlet positions. Results of their study highlighted the strong influence in the mixing process of the car immediately behind the emitted plume. The focus of their study, however, was on internal air quality rather than on the dispersion process in the near-wake.

Richards (2002) and Baker (2001) analysed flow and dispersion characteristics in the near-wake of a vehicle model with no cross-wind. Results from these wind tunnel studies highlighted the close relationship between the inert tracer concentration field and the velocity and turbulence fields. Concentration fluctuations, measured by a flame ionisation detector (FID), were consistent with the fluctuations in the velocity 
field (obtained through a combination of particle image velocimetry, PIV, hot-wire anemometry, HWA and flow visualisation methods). The time histories of concentration had a "peaky", intermittent nature. Kanda et al. (2006a) measured the flow characteristics in the near-wake of small-scale models of a car and a lorry emitting a thermally buoyant plume. They used PIV and laser Doppler anemometry (LDA) to measure velocity and turbulence fields in the wind tunnel, and a FID to measure tracer gas concentrations. They found that the buoyancy of the exhaust had generally a minor effect on the dispersion behaviour. The results from this study were then used as a basis for studying multi-vehicle configurations (Kanda et al., 2006b), with an approach similar to the one adopted by Clifford et al. (1997) in the wind tunnel.

The effect of cross-winds on a single wake generated by a model of lorry was studied by Baker and Hargreaves (2001). The model was placed on a moving model rig and was propelled across a wind tunnel in which an atmospheric boundary layer had been simulated. Experiments were carried out with both open country and with a street canyon wind simulations.

\subsection{Discussion}

In order to improve our understanding of the flow and mixing processes in the near-wake, and also in the far-wake, more studies on the basic configuration of the wake from a single vehicle are needed. As stated above, most of the previous studies dealt with more complex situations such as multiple vehicles, cross-winds and complex geometry (for example, street canyons). They are very useful for deriving parameterisations of the traffic flow for use in larger scale models, but cannot be used to characterise the single wake. The few studies specifically designed to study a single 
wake, and the near-wake in particular (Baker, 2001; Richards, 2002; Kanda et al., 2006a), have highlighted the differences in the plume behaviour depending on the shape of the model and the boundary conditions, so more experimental studies are needed to generalise their findings.

A possible improvement on past experimental studies would be the use of a rolling floor in the wind tunnel. All the above mentioned studies suffered from the presence of a boundary layer developing close to the ground, which can interfere with a developing wake flow field and its measurement. Kanda et al. (2006a) tried to prevent this problem by placing the model on an elevated table; nonetheless, a boundary layer grows on the table top and reaches about $3 \mathrm{~cm}$ thick at about $1 \mathrm{~m}$ from the upwind edge of the table, resulting in unrealistic velocity and turbulence fields (Kanda et al., 2006a). Baker and Hargreaves (2001) solved the problem by using a ballistic model within the wind tunnel.

In order to develop parameterised models specifically designed for nanoparticle dispersion there is first a need to understand sufficiently the dispersion of inert gases. However, as stated at the beginning of this section, measurements of nanoparticle number concentrations in the wind tunnel, to be possibly compared with field measurements and numerical simulations, will add valuable information.. In this case, a few problems need to be overcome to model particle dispersion properly. In particular, the presence of particle elements in the emitted plume adds further scaling conditions to have similarity with the full scale situation. For example, see discussions on similarity criteria in Kind (1986), Xuan and Robins (1994), Goossens and Offer (1990) and Parker and Kinnersley (2004). The use of larger scale models is essential 
to meet the similarity criteria, besides providing measurements with better spatial resolution.

\section{Mathematical modelling}

As highlighted in the previous sections, further insight is needed on the transformation processes and dynamics of nanoparticles to develop reliable mathematical models for the estimation of their number concentrations in vehicle wakes. This section provides an overview of approaches for modelling the dispersion of pollutants in the wakes of moving vehicles, starting with the most recent advances in fully computational models (CFD approach). These have their limitations due to their complex nature and their requirement of intensive computational resources. The main objective of this section is to discuss possible approaches for fast parametric models that could be used as stand alone or as sub-modules within a multiscale air quality model for routine air quality assessment and forecasts A simple mathematical approach, which needs further development, is proposed as an example of such approaches.

\subsection{CFD modelling}

With recent advances in computational modelling, it has become possible to investigate flow and dispersion characteristics in the wakes of vehicles by using a numerical approach, CFD. However, such methods cannot be used for operational modelling (e.g., real time calculations, forecasting, long-term scenario analysis for air quality assessment) because of their resource demands, though but they can be used, like experiments, to gain insight on the phenomena involved, especially when integrated with experimental techniques. 
As already discussed in Section 2.1, a number of approaches to CFD modelling of gaseous pollutants in the wakes of vehicles has been attempted. These include Reynolds-averaged Navier-Stokes (RANS) simulations (using the $k-\varepsilon$, RNG $k-\varepsilon$, or $k$ $\varepsilon /$ Chen turbulence models; Richards, 2002; Richards et al., 2000; Kim et al., 2001), or large eddy simulations (LES; Dong and Chan, 2006; Chan et al., 2008). The latter approach is more resource-intensive but has a better potential for unsteady applications such as the dispersion of nanoparticles in vehicle wakes.

The evolution of nanoparticles can be implemented in numerical models by using the general dynamic equation (GDE; Friedlander, 2000). This equation cannot easily be solved, and several numerical techniques have been developed for this purpose, including sectional methods (Garrick et al., 2006) and the method of moments (McGraw et al., 1998). A recent successful application of such methods was carried out by Chan et al. (2010); using an LES approach for turbulence, coupled with a dispersion model based on the direct quadrature method of moments (DQMOM). Their results offer an interesting analysis of the time scales of the processes involved in nanoparticle evolution, yielding conclusions similar to those reported in Section 3 (see also Table 2), demonstrating the potential of the numerical approach.

A different numerical technique was adopted by Albriet et al. (2010). They coupled a CFD RANS code (using the $k-\varepsilon$ turbulence model) with a box modelling approach for aerosol dynamics (in particular they adopted the Modal Aerosol Model, MAM). Comparisons of the numerical results with measured values were promising, though the authors pointed out the necessity of including the vehicle wake for a more correct characterisation. This may prove to be difficult with the RANS approach, while LES models may be more suitable for this purpose. 
CFD models have proved to be valuable tools for nanoparticle dispersion investigations. However, despite the fast development of numerical techniques and exponential increasing in computational resources, the operational use of such models is still very limited and likely to remain so. Routine air quality assessment, including air quality forecasting and long-term scenario analysis, need faster approaches; thus simplified parameterised models are still necessary.

\subsection{Dispersion of pollutants in vehicle wake}

Generally, the dispersion of pollutants in vehicle wakes in parametric models is addressed through Gaussian dispersion theory and represents the traffic flow by stationary point and line sources placed at intervals along a road, rather than considering a moving point source (Eskridge and Hunt, 1979; Baker, 1996; 2001). Dispersion models that include details of the vehicle wake flow (either near or far wake) are less common. The few models that consider such effects are primarily based on the wake descriptions developed by Eskridge and Hunt (1979), and successive modifications (Eskridge et al., 1979; Eskridge and Rao, 1983; Eskridge and Rao, 1986; Baker, 1996; Hider et al., 1997) Hargreaves and Baker, 1997). These models are only valid at a non-dimensional distance from the vehicle of about $X / h \geq$ 15 (where $X$ is the longitudinal distance from the rear of the vehicle, and $h$ is the vehicle height). The well known ROADWAY model uses surface similarity theory and the wake theory of Eskridge and Hunt (1979) to determine the atmospheric structure along a roadway due to the presence of vehicles (Eskridge et al., 1979; Eskridge and Rao, 1983; Eskridge and Rao, 1986). A conservation of species or advection-diffusion equation is solved using finite difference approximations to obtain the concentration field for multi-vehicle emissions. The model is essentially designed to predict vertical and lateral concentration distributions near roadways. As 
pointed out in Section 2.2, the theory of Eskridge and Hunt (1979) and subsequent developments by Hider et al. (1997) only describe the velocity and turbulence fields in the far wake. While the overall velocity deficit caused by the near wake is included as a boundary condition at $X=15 \mathrm{~h}$, the theories do not include detailed modelling of the near wake region and ignore any interaction between the longitudinal vortices and the drag induced wake.

\subsection{Approaches derived from building wakes}

In contrast with the rare attempts at modelling dispersion in vehicle wake, extensive work has been carried out for characterising and simulating the dispersion phenomena in the wake of buildings. For example, see the reviews by Canepa (2004), Walsh and Jones (2002) and Olesen and Genikhovich (2000). The physical processes involved are similar to those in vehicle wakes in many respects and a similar approach can be adopted.

One of the most advanced models for treating dispersion in the wake of buildings under steady wind conditions is ADMS-BUILD which is included in the ADMS model. A very similar approach is adopted by another widely used model called (PRIME i.e. Plume Rise Model Enhancements) which is developed by the United States Environmental Protection Agency and Electric Power Research Institute (Schulman et al., 2000). The ADMS 'Building Effects Module' computes the dispersion of pollution from sources near isolated large buildings or an effective building representing a group of closely spaced buildings. The model is based on that of Hunt and Robins (1982) and is described in more detail by Apsley (1988) and Robins et al. (1997). The model assumes a simplified flow field that is based on a well mixed cavity (or recirculating flow region, correspondent to the near-wake) and a 
downstream turbulent wake (the far or main wake; see Section 2). It takes into account different source positions (within and out of the near wake) and allows for complete or partial entrainment into the recirculating flow region. Within the near wake region concentrations are assumed to be uniform due to rapid turbulent mixing. For partially entrained plumes, the entrained and non-entrained components form a two-plume structure downwind (in the far wake).

Starting from the above modelling approaches, a simple model can be built for modelling pollutant concentrations in vehicle wakes with the integrated use of the situation shown in Fig. 3 and building wake theory. For instance, consider a vehicle with height $h$ moving at speed $V$ into a weak head wind $U$ (i.e. $V>>U$ ). The standard building wake theory assumes a well mixed near wake region having a volume $\alpha h^{3}$ and an average exchange velocity $v^{\prime}$ operating over the surface area of the near-wake $\gamma^{\prime} h^{2}$ (where $\alpha$ and $\gamma^{\prime}$ are constants taking into account the shape of the near wake). Then the change in pollutant mass per unit time can be expressed as:

$$
\frac{\mathrm{d} m}{\mathrm{~d} t}=\frac{\mathrm{d}}{\mathrm{d} t}\left(\alpha h^{3} C_{w}\right)=-q=-\gamma^{\prime} h^{2} v^{\prime} C_{w}
$$

where $m$ is the pollutant mass in the near-wake, $C_{w}$ the mean concentration within the near-wake and $q$ the mass flux of pollutant from the near wake. Since $V>>U, v^{\prime}$ can be assumed to be proportional to the vehicle speed $(V)$ and Eq. (1) can be modified to:

$$
\frac{\mathrm{d} C_{w}}{\mathrm{~d} t}=-\frac{2 h^{2} V C_{w}}{\alpha h^{3}}=-\frac{C_{w}}{\beta(h / V)}=-\frac{C_{W}}{T_{W}}
$$

where $\gamma V=\gamma^{\prime} v^{\prime}$ (being $\gamma$ another constant), and $T_{w}$ is the time scale of pollutant decay, defined as $T_{w}=\beta h / V$; the empirical constant $\beta$ can be taken as $\approx 5$ (Fackrell, 1984). Equation (2) can be integrated as: 


$$
C_{W}=C_{0} \exp \left(-\frac{t}{T_{w}}\right)=C_{0} \exp \left(-\frac{x}{\beta h}\right)
$$

where $x$ is the distance travelled by the vehicle at time $t$. Equation (3) shows that material is spread by the moving vehicle over a length of road of several times $\beta h$. Taking into account a background concentration $C(x)$, and assuming it uniform along the $y$ and $z$ axes (see Fig. 3), Eq. 2 can be rewritten as:

$$
\frac{\mathrm{d} C_{w}}{\mathrm{~d} t}=-\frac{C_{W}-C}{T_{W}}
$$

and then:

$$
\frac{\mathrm{d} C_{w}}{\mathrm{~d} x}=-\frac{C_{W}-C}{V T_{W}}=-\frac{C_{W}-C}{\beta h}
$$

leading to:

$$
q(x)=-\frac{\mathrm{d} m}{\mathrm{~d} x}=-\alpha h^{3} \frac{\mathrm{d} C_{w}}{\mathrm{~d} x}=\frac{\alpha h^{2}\left(C_{w}-C\right)}{\beta}
$$

where $q(x)$ is the loss rate of pollutant from the wake as the vehicle move along $x$. This value can be then incorporated as a source term for standard plume models (e.g., a Gaussian model, taking into account multiple vehicles moving along $x$ and calculating the emission per line length, or a box model to be used within a street canyon).

Note that this is a very simple formulation that considers only the inert pollutants in the near wake of a moving vehicle in calm or light wind conditions. It needs to be further refined and validated for the prediction of nanoparticles number concentrations and size distributions after taking into account particle transformation processes. The proposed model also has its limitations, such as assuming concentrations in the near-wake are uniform, as is also the case in previously developed building effect models (e.g. ADMS-BUILD). Nevertheless, it is a useful 
basis that the authors intend to develop further for nanoparticle dispersion, following results from ongoing field and wind tunnel studies (see Section 7).

\subsection{Discussion}

A number of modelling approaches have been attempted in recent years for short range dispersion of nanoparticles and, more generally, aerosols (Pohjola et al., 2003; Zhang and Wexler, 2004; Zhang et al., 2004; Pohjola et al., 2006). However, they do not include any modifications due to the effect of vehicle wakes. Furthermore, methods for calculating nanoparticle number concentrations in the wake of a moving vehicle have not yet even been proposed.

Given the importance of the interactions between nanoparticle transformation processes in the early stages after the emission and the flow and turbulence fields in the near wake, a way of integrating the two approaches must be found. A potential methodology to develop this topic further could come from the approach outlined in subsection 6.2, derived from extensive work on building wakes. However, further research is needed for adapting this simple approach to the particular characteristics of vehicle wakes, such as the flow below the car (unlike buildings), the emission source located within (or close to) the near-wake, the streamlined shape of the vehicles (and the differences between them). The near-wake dispersion process should also be modelled in more detail if a useful nanoparticle dispersion model is eventually to be developed.

\section{Conclusions and future work}

This article provides an overview of significant past research work relevant to modelling the dispersion of pollutants in the wake of vehicles, with a particular focus on nanoparticle dispersion. Literature related to vehicle wakes and nanoparticle 
dispersion has been reviewed, taking into account field measurements, wind tunnel experiments and mathematical modelling approaches.

Field measurements and nanoparticle dynamics modelling studies highlighted the very short time scales associated with nanoparticle transformations in the earliest stages after emission. These transformations are strongly dependent on the flow and turbulence conditions immediately behind the emitting vehicle, hence the need to characterise these in some detail, especially in the near wake. This is particularly important when attention is focussed on short range (i.e. street scale) dispersion. Very few studies have addresses this topic and those that do are generally applicable only in the far wake. A simple approach derived from building wake studies has also been proposed. Given the lack of experimental studies that could be analysed to determine suitable parameterisations, further research is needed in the areas of wake dispersion and nanoparticle dynamics.

Field experiments purposely designed for measuring nanoparticle evolution in the wakes of vehicles are rare; there is a clear research need involving fast response measurements that can provide detailed information on transformation processes occurring just after the release of emissions as a result of interactions with the near wake flow and turbulence.

Wind tunnel experiments for characterising vehicle wakes have successfully been used in the past in the few studies on this topic that can be found in the literature. The methodologies highlighted in those works may be further improved and applied specifically for the development of nanoparticle dispersion models in the wake of vehicles. One of the main findings of previous wind tunnel studies is the strong dependence of the near wake characteristics on the vehicle shape, so there is a clear 
necessity of studying different geometries in order to generalise the results to be used for model development. None of the previous laboratory studies on pollutant dispersion in vehicle wakes involved the use of a rolling floor (although other solutions to the boundary layer development problem exist, as reviewed in Section 5.3); this technique is widely used in aerodynamic studies and it is essential to reproduce realistic flow and turbulence fields in the wind tunnel. Nanoparticle concentration measurements in the wind tunnel have never been attempted; these would greatly help characterise the dispersion process, though care must be given to the key similarity criteria in reduced scale models.

As there are several unanswered questions relating to particle dynamics, dispersion and modelling at this very fine scale, firm scientific results from ongoing research will provide a sound basis for understanding this topic in a more comprehensive manner. Future work on this topic should address as a priority the following steps:

- real-time measurements of particle number distributions at various spatial locations in the wake of vehicles during field experiments;

- integration of these measurement by extensive wind tunnel experiments allowing aerodynamic studies in realistic conditions with the use of a rolling floor;

- development of a mathematical model for the dispersion of nanoparticles number and size distributions in vehicle wake (e.g., using the approach proposed in Section 6.2) using the key findings from the field and wind tunnel experiments.

Such work is underway at EnFlo, University of Surrey. The project, funded by the EPSRC, will include field measurements using the recently acquired DMS50 
nanoparticle spectrometer, and wind tunnel experiments in the newly built aerodynamic facility at the EnFlo lab. The project will end in July 2011.

\section{Acknowledgements}

This work has been carried out as a part of the EPSRC grant EP/H026290/1 which focuses on the dispersion of nanoparticles in the wake of vehicles. PK gratefully acknowledges the receipt of this grant which support his research on atmospheric nanoparticles.

\section{References}

Ahmad, K., Khare, M., Chaudhry, K.K., 2002. Model vehicle movement system in wind tunnels for exhaust dispersion studies under various urban street configurations,. Journal of Wind Engineering and Industrail Aerodynamics 90, 1051-1064.

Ahmed, S., 1981. An experimental study of the wake structures of typical automobile shapes. Journal of Wind Engineering and Industrial Aerodynamics 9, 49-62.

Albriet B., Sartelet K.N., Lacour S., Carissimo B., Seigneur C., 2010. Modelling aerosol number distributions from a vehicle exhaust with an aerosol CFD model. Atmospheric Environment 44, 1126-1137.

Apsley, D.D., 1988. A model for dispersion in the wake of large buildings. CEGB Report RD/L/3359/R88.

AQEG, 2005. Particulate matter in the United Kingdom, AQEG. Defra London.

Baker, C.J., 1996. Outline of a novel method for the prediction of atmospheric pollution dispersal from road vehicles. Journal of Wind Engineering and Industrial Aerodynamics 65, 395-404.

Baker, C.J., 2001. Flow and dispersion in ground vehicle wakes. Journal of Fluids and Structures 15, 1031-1060.

Baker, C.J., Hargreaves, D.M., 2001. Wind tunnel evaluation of a vehicle pollution dispersion model. Journal of Wind Engineering and Industrial Aerodynamics 89, 187-200.

Benson, P.E., 1989. CALINE4 - A dispersion model for predicting air pollutant concentrations near roadways. California Department of Transport, Technical Report FHWA/CA/TL-84/15.

Berkowicz, R., 2000. Operational street pollution model-a parameterized street pollution model. Environmental Monitoring and Assessment 65, 323-331.

Brugge, D., Durant, J.L., Rioux, C., 2007. Near-highway pollutants in motor vehicle exhaust: A review of epidemiologic evidence of cardiac and pulmonary health risks. Environmental Health 6, 23. 
Canepa, E., 2004. An overview about the study of downwash effects on dispersion of airborne pollutants. Environmental Modelling \& Software 19, 1077-1087.

CERC., 2006. ADMS-Urban, an urban air quality management system. User guide version 2.2 Cambridge, UK.

Chan T.L., Liu Y.H., Chan C.K., 2010. Direct quadrature method of moments for the exhaust particle formation and evolution in the wake of the studied ground vehicle. Journal of Aerosol Science 41, 553-568.

Chan, T.L., Luo, D.D., Cheung, C.S., Chan, C.K., 2008. Large eddy simulation of flow structures and pollutant dispersion in the near-wake region of the studied ground vehicle for different driving conditions. Atmospheric Environment 42, 5317-5339.

Charron, A., Birmili, W., Harrison, R.M., 2008. Fingerprinting particle origins according to their size distribution at a UK rural site. Journal of Geophysical Research 113, DO7202, doi:7210.1029/2007JD008562, 002008.

Charron, A., Harrison, R.M., 2003. Primary particle formation from vehicle emissions during exhaust dilution in the road side atmosphere. Atmospheric Environment 37, 4109-4119.

Clifford, M.J., Clarke, R., Riffat, S.B., 1997. Local aspects of vehicular pollution. Atmospheric Environment 31, 271-276.

Davidson, C., Phalen, R., Solomon, P., 2005. Airborne particulate matter and human health: a review. Aerosol Science and Technology 39, 737-749.

Di Sabatino, S., Kastner-Klein, P., Berkowicz, R., Britter, R.E., Fedorovich, E., 2003. The modelling of turbulence from traffic in urban dispersion models - part I: theoretical considerations. Environmental Fluid Mechanics 3, 129-143.

Donaldson, K., Tran, L., Albert Jimenez, L.A., Duffin, R., Newby, D.E., Mills, N., MacNee, W., Stone, V., 2005. Combustion-derived nanoparticles: A review of their toxicology following inhalation exposure. Particle \& Fibre Toxicology 5/6, 553-560.

Dong, G., Chan, T.L., 2006. Large eddy simulation of flow structures and pollutant dispersion in the near-wake region of a light-duty diesel vehicle. Atmospheric Environment 40, 1104-1116.

Eskridge, R.E., Binkowski, F.S., Hunt, J.C.R., Clark, T.L., Demerijan, K.L., 1979. Highway modelling Part II: advection of SF6 tracer gas. Journal of Applied Meteorology 18, 401-412.

Eskridge, R.E., Hunt, J.C.R., 1979. Highway modelling Part I: prediction of velocity and turbulence fields in the wake of vehicles. Journal of Applied Meteorology 18, 387-400.

Eskridge, R.E., Rao, S.T., 1983. Journal of Climate and Applied Meteorology. Measurement and prediction of traffic- induced turbulence and velocity fields near roadways 22, 1431-1443.

Eskridge, R.E., Rao, S.T., 1986. Turbulent diffusion behind vehicles: experimentally determined turbulence mixing parameters. Atmospheric Environment 20, 851860 .

Eskridge, R.E., Thompson, R.S., 1982. Experimental and theoretical study of the wake of a block-shaped vehicle in a shear-free boundary flow. Atmospheric Environment - Part A General Topics 16, 2821-2836. 
Fackrell, J.E., 1984. Parameters characterising dispersion in the near wake of a building. Journal of Wind Engineering and Industrial Aerodynamics 16, 97-118.

Friedlander, S.K., 2000. Smoke, dust and haze: Fundamentals of aerosol dynamics. Oxford: Oxford University Press, Inc.

Garrick, S.C., Lehtinen, K.E.J., Zachariah, M.R., 2006. Nanoparticle coagulation via a Navier-Stokes/nodal methodology: Evolution of the particle field. Journal of Aerosol Science 37, 555-576.

Gidhagen, L., Johansson, C., Langner, J., Olivares, G., 2004. Simulation of NOx and ultrafine particles in a street canyon in Stockholm, Sweden. Atmospheric Environment 38, 2029-2044.

Goossens, D., Offer, Z.I., 1990. A previous termwind tunnelnext term simulation and field verification of desert dust deposition (Avdat Experimental Station, Negev Desert). Sedimentology 37, 7-22.

Hackett, J.E., Williams, J.E., Baker, J.B., Wallis, S.B., 1987. On the influence of ground movement and wheel rotation in tests on modern car shapes. SAE Technical Paper Series Technical Report 870245.

Hargreaves, D.M., Baker, C.J., 1997. Gaussian puff model of an urban street canyon. Journal of Wind Engineering and Industrial Aerodynamics 69-71, 927-939

Hider, Z.E., Hibberd, S., Baker, C.J., 1997. Modelling particulate dispersion in the wake of vehicle. Journal of Wind Engineering and Industrial Aerodynamics 67\&69, 733-744.

Hucho, W.-H., 1987. Aerodynamics of road vehicles. SAE Technical paper ISBN, 07680-0029-7.

Hunt, J.C.R., Robins, A.G., 1982. A model for assessing dispersion of plumes from sources in the vicinity of cuboid shaped buildings. Proceedings of the EUROMECH Conference on Surface Mounted Bluff Bodies in Turbulent Boundary Layers, Lisbon.

ICRP, 1994. ICRP Publication 66: Human respiratory tract model for radiological protection A Report of a task group of the International Commission on Radiological Protection, 1-482.

Jacobson, M.Z., 2005. Fundamentals of atmospheric modeling, 2nd Edition ed. Cambridge University Press.

Johansson, C., Norman, M., Gidhagen, L., 2007. Spatial \& temporal variations of PM10 and particle number concentrations in urban air. Environmental Monitoring and Assessment 127, 477-487.

Kanda, I., Uehara, K., Yamao, Y., Yoshikawa, Y., Morikawa, T., 2006a. A windtunnel study on exhaust gas dispersion from road vehicles--Part I: Velocity and concentration fields behind single vehicles. Journal of Wind Engineering and Industrial Aerodynamics 94, 639-658.

Kanda, I., Uehara, K., Yamao, Y., Yoshiwara, Y., Morikawa, T., 2006b. A windtunnel study on exhaust-gas dispersion from road vehicles-Part II: Effect of vehicle queues. Journal of Wind Engineering and Industrial Aerodynamics 94 659-673.

Kastner-Klein, P., Berkowicz, R., Fedorovich, E., 2001a. Evaluation of scaling concepts for traffic-produced turbulence based on laboratory and full-scale concentration measurements in street canyons. In: Proceedings of the Third 
International Conference on Urban Air Quality 19-23 March 2001, Loutraki, Greece.

Kastner-Klein, P., Berkowicz, R., Plate, E.J., 2000a. Modelling of vehicle induced turbulence in air pollution studies for streets. International Journal of Environment and Pollution 14, 496-507.

Kastner-Klein, P., Fedorovich, E., Ketzel, M., Berkowicz, R., Britter, R., 2003. The modelling of turbulence from traffic in urban dispersion models - Part II: evaluation against laboratory and full-scale concentration measurements in street canyons. Environmental Fluid Mechanics 3, 145-172.

Kastner-Klein, P., Fedorovich, E., Rotach, M., 2001b. A wind tunnel study of organised and turbulent air motions in urban street canyons. Journal of Wind Engineering and Industrial Aerodynamics 89, 849-861.

Kastner-Klein, P., Fedorovich, E., Sini, J.-F., Mestayer, P.G., 2000b. Experimental and nu- merical verification of similarity concept for diffusion of car exhaust gases in urban street canyons. Environmental Monitoring and Assessment 65, 353-361.

Ketzel, M., Berkowicz, R., 2004. Modelling the fate of ultrafine particles from exhaust pipe to rural background: an analysis of time scales for dilution, coagulation and deposition. Atmospheric Environment 38, 2639-2652.

Khare, M., Chaudhry, K.K., Gowda, R.M.M., Ahmad, K., 2002. Heterogeneous traffic induced effects on vertical dispersion parameter in the near field of roadways-a wind tunnel study. Environmental Modeling and Assessment 7, 915.

Kim, D.H., Gautam, M., Gera, D., 2001. On the prediction of concentration variations in a dispersing heavy-duty truck exhaust plume using k-epsilon turbulent closure. Atmospheric Environment 35, 5267-5275.

Kind, R.J., 1986. Snowdrifting: a review of previous termmodellingnext term methods. Cold Regions Science and Technology 12, 217-228.

Kittelson, D.B., Watts, W.F., Johnson, J.P., 2006a. On-road and laboratory evaluation of combustion aerosols - Part 1 : Summary of deisel engine results. Journal of Aerosol Science 37, 913-930.

Kumar, P., Fennell, P., Britter, R., 2008a. Effect of wind direction and speed on the dispersion of nucleation and accumulation mode particles in an urban street canyon. Science of the Total Environment 402, 82-94.

Kumar, P., Fennell, P., Britter, R., 2008b. Measurements of particles in the 5-1000 nm range close to road level in an urban street canyon. Science of the Total Environment 390, 437-447.

Kumar, P., Fennell, P., Langley, D., Britter, R., 2008c. Pseudo-simultaneous measurements for the vertical variation of coarse, fine and ultra fine particles in an urban street canyon. Atmospheric Environment 42, 4304-4319.

Kumar, P., Fennell, P., Symonds, J., Britter, R., 2008d. Treatment of losses of ultrafine aerosol particles in long cylindrical sampling tubes during ambient measurements. Atmospheric Environment 42, 8819-8826.

Kumar, P., Fennell, P., Hayhurst, A., Britter, R.E., 2009a. Street versus rooftop level concentrations of fine particles in a Cambridge street canyon. Boundary-Layer Meteorology 131, 3-18. 
Kumar, P., Garmory, A., Ketzel, M., Berkowicz, R., Britter, R., 2009b. Comparative study of measured and modelled number concentrations of nanoparticles in an urban street canyon. Atmospheric Environment 43, 949-958.

Kumar, P., Robins, A., Britter, R., 2009c. Fast response measurements for the dispersion of nanoparticles in a vehicle wake and in a street canyon. Atmospheric Environment 43, 6110-6118.

Kumar, P., Fennell, P., Robins, A., 2010a. Comparison of the behaviour of manufactured and other airborne nanoparticles and the consequences for prioritising research and regulation activities. Journal of Nanoparticle Research $12,1523-1530$

Kumar, P., Robins, A., Vardoulakis, S., Britter, R., 2010b. A review of the characteristics of nanoparticles in the urban atmosphere and the prospects for developing regulatory control. Atmospheric Environment in press, doi:10.1016/j.atmosenv.2010.08.016.

Kumar, P., Robins, A., ApSimon, H., 2010c. Nanoparticle emissions from biofuelled vehicles - their charcterstics and impact on the number-based regulation of atmospheric particles. Atmospheric Science Letters in press, doi:10.1002/asl.1307.

Longley, I.D., Gallagher, M.W., Dorsey, J.R., Flynn, M., Allan, J.D., Alfarra, D., Inglish, D., 2003. A case study of aerosol $(4.6 \mathrm{~nm}<\mathrm{Dp}<10 \mu \mathrm{m})$ number and mass size distribution measurements in a busy street canyon in Manchester, U.K. Atmospheric Environment 37, 1563-1571.

McGraw, R., Nemesure, S., Schwartz, S.E., 1998. Properties and evolution of aerosols with size distributions having identical moments. Journal of Aerosol Science 29, 761-772.

Oberdorster, G., 2000. Toxicology of ultrafine particles: in vivo studies. Philosophical Transactions of the Royal Society of London A 358, 2719-2740.

Olesen, H.R., Genikhovich, E., 2000. Building downwash algorithm for the OML atmospheric dispersion model. National Environmental Research Institute, Denmark Research Notes from NERI No. 123, 30 pp.

Parker, S.T., Kinnersley, R.P., 2004. A computational and wind tunnel study of particle dry deposition in complex topography. Atmospheric Environment 38, 3867-3878.

Pey, J., Querol, X., Alastuey, A., Rodríguez, S., Putaud, J. P., Van Dingenen, R., 2009. Source apportionment of urban fine and ultra-fine particle number concentration in a Western Mediterranean city. Atmospheric Environment 43, 4407-4415.

Pohjola, M., Pirjola, L., Kukkonen, J., Kulmala, M., 2003. Modelling of the influence of aerosol processes for the dispersion of vehicular exhaust plumes in street environment. Atmospheric Environment 37, 339-351.

Pohjola, M.A., Pirjola, L., Kukkonen, J., Kulmala, M., 2006. Correction to modelling of the influence of aerosol processes for the dispersion of vehicular exhaust plumes in street environment. Atmospheric Environment 40, 311-314.

Richards, K.A., 2002. Computational modelling of pollution dispersion in the near wake of a vehicle. University of Nottingham $\mathrm{PhD}$ Thesis. 
Richards, K.A., Wright, N.G., Baker, C.J., Baxendale, A., 2000. Computational modelling of pollutant dispersion in near wake of vehicles. Proceedings of CWE2000 Conference, Birmingham, UK.

Robins, A.G., McHugh, C.A., Carruthers, D.J., 1997. Testing and evaluating the ADMS building effects module. International Journal of Environment and Pollution 8, 708-717.

Schulman, L.L., Strimaitis, D.G., Scire, J.S., 2000. Development and evaluation of the PRIME plume rise and building downwash model. Journal of Air Waste Management Association 50, 378-390.

Shi, P.J., Khan, A.A., Harrison, R.M., 1999. Measurements of ultra fine particle concentration and size distribution in the urban atmosphere. The Science of the Total Environment 235, 51-64.

Walsh, C., Jones, J.A., 2002. Atmospheric dispersion from releases in the vicinity of buildings. National Radiological Protection Board, Report NRPB-W16.

Weber, S., Kuttler, W., Weber, K., 2006. Flow characteristics and particle mass and number concentration variability within a busy street canyon. Atmospheric Environment 40, 7565-7578.

Wehner, B., Wiedensohler, A., 2003. Long term measurements of submicrometer urban aerosols: statistical analysis for correlations with meteorological conditions and trace gases. Atmospheric Chemistry Physics 3, 867-879.

Xuan, J., Robins, A., 1994. The effects of turbulence and complex terrain on dust emissions and depositions from coal stockpiles. Atmospheric Environment 28, 1951-1960.

Yamartino, R.J., Wiegand, G., 1986. Development and evaluation of simple models for the flow, turbulence and pollutant concentration fields within an urban street canyon. Atmospheric Environment 20, 2137-2156.

Yao, X.H., Lau, N.T., Fang, M., Chan, C.K., 2006. On the time-averaging of ultrafine particle number size spectra in vehicular plumes. Atmospheric Chemistry and Physics 6, 4801-4807.

Zhang, K.M., Wexler, A.S., 2004. Evolution of particle number distribution near roadways - Part I: analysis of aerosol dynamics and its implications for engine emission measurement. Atmospheric Environment 38, 6643-6653.

Zhang, K.M., Wexler, A.S., Zhu, Y.F., Hinds, W.C., Sioutas, C., 2004. Evolution of particle number distribution near roadways - Part II: the 'Road-to-Ambient' process. Atmospheric Environment 38, 6655-6665. 


\section{List of Figures}

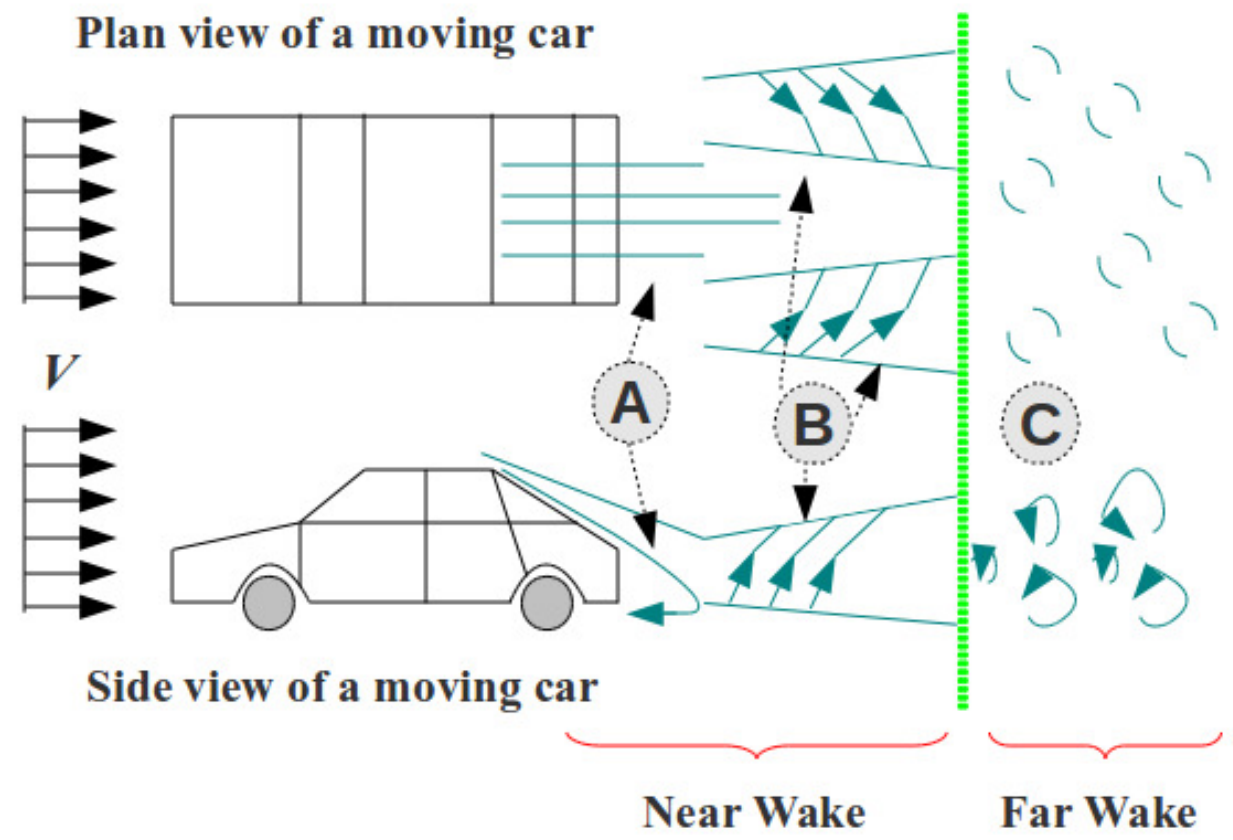

Fig. 1. Definition of near and main/far wake regions; A: near wake, flow reversal; B: near wake, longitudinal vortices; C: far wake, general turbulence, no discernible flow structure; adapted from Hucho (1987). 


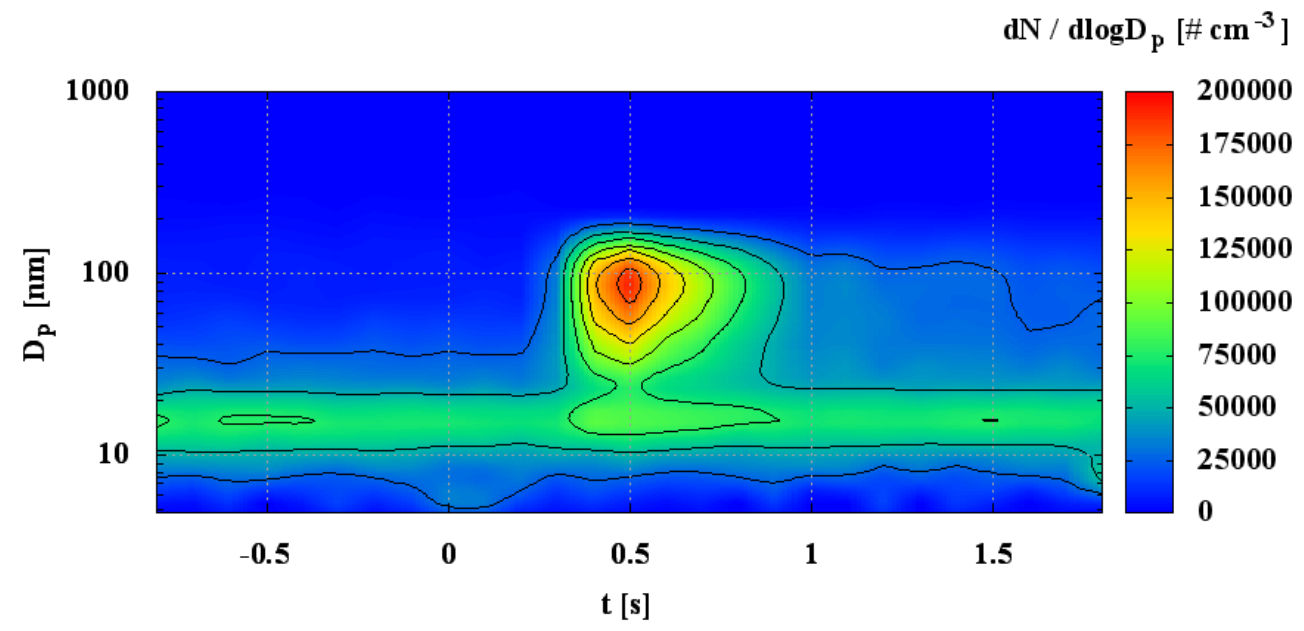

Fig. 2. Temporal evolution of the particle number distribution (PND) due to emissions from a single diesel-engined car during the measurements performed by Kumar et al. (2009c).

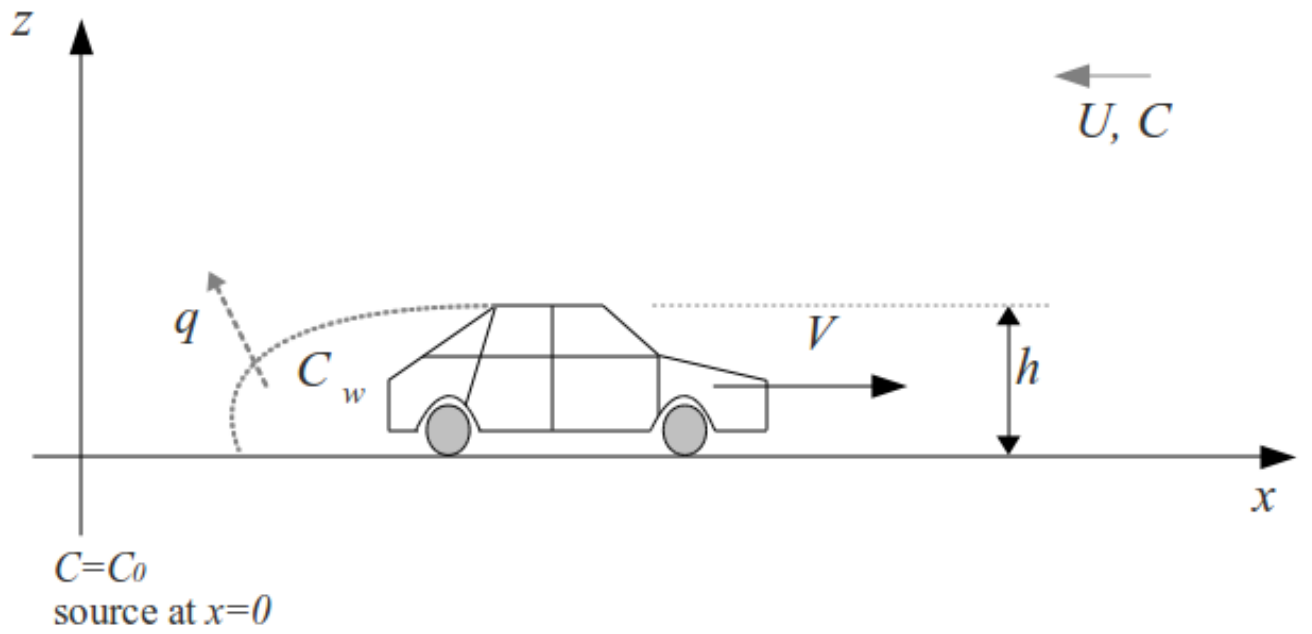

Fig . 3. Schematic of a simple vehicle wake dispersion model. 


\section{List of Tables}

Table 1. Summary of the key flow and mixing characteristics in near and far/main wake regions of moving vehicles.

\begin{tabular}{ll}
\hline Wake regions & Key flow and mixing features \\
\hline Near wake & Flow is very complex in near wake region compared with \\
main wake region \\
- Vehicle induced turbulence is intense in near wake and \\
dominate the mixing \\
- A detailed characterisation is necessary when short time \\
scales in the evolution of the considered pollutant are \\
applicable \\
- Atmospheric turbulence (mechanically or thermally \\
generated) lead the further mixing in far wake \\
- Interaction between wakes from several vehicles should be \\
considered \\
- The dispersion process is strongly affected by the local \\
geometry (terrain and/or canyon effect)
\end{tabular}


Table 2. Relative importance of different nanoparticle transformation processes.

\begin{tabular}{ll}
\hline Mechanism & Key features \\
\hline Dilution & - Dependent on background concentration levels \\
- Fastest transformation process \\
- Strongly affected by the flow and turbulence fields in vehicle \\
wakes \\
\hline - Active in the first stages after the emission \\
- Terminated by dilution of the participating gases below their \\
saturation ratio \\
\hline - It does not change the particle number concentration, but it \\
- Time scale dependent on growth rate, can vary from the \\
coagulation/deposition values to dilution values \\
- Time scale inversely proportional to particle number \\
concentration \\
- It does not play a significant role compared to dilution and, \\
usually, condensation \\
- It may become relevant in particular geometrical situations \\
(e.g. confined spaces such as tunnels, etc.)
\end{tabular}

- Wet deposition: occurring in presence of rain 